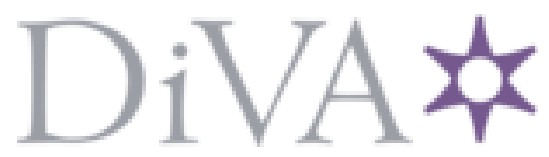

http://www.diva-portal.org

\title{
Postprint
}

This is the accepted version of a paper presented at IEEE Information Theory Workshop, ITW 2012; Lausanne; 3 September 2012 through 7 September 2012.

Citation for the original published paper:

Du, J., Xiao, M., Skoglund, M., Shamai (Shitz), S. (2012)

Short-Message Noisy Network Coding with Partial Source Cooperation.

In: Information Theory Workshop (ITW), 2012 IEEE (pp. 144-147). IEEE

http://dx.doi.org/10.1109/ITW.2012.6404644

N.B. When citing this work, cite the original published paper.

Permanent link to this version:

http://urn.kb.se/resolve?urn=urn:nbn:se:kth:diva-98095 


\section{Short-Message Noisy Network Coding with Partial Source Cooperation}

\author{
Jinfeng Du, Ming Xiao, Mikael Skoglund \\ ACCESS Linnaeus Center \\ Royal Institute of Technology \\ Stockholm 10044, Sweden \\ Email: jinfeng@kth.se, \{ming.xiao, skoglund\}@ee.kth.se
}

\author{
Shlomo Shamai (Shitz) \\ Department of Electrical Engineering \\ Technion-Israel Institute of Technology \\ Technion City, Haifa 32000, Israel \\ Email:sshlomo@ee.technion.ac.il
}

\begin{abstract}
Noisy network coding (NNC) has been shown to outperform standard compress-and-forward (CF) in networks with multiple relays and/or multiple destinations. Recently, shortmessage noisy network coding (SNNC) has been proved to achieve the same rate region as NNC for independent sources but with significantly reduced encoding delay and decoding complexity. In this paper, we show that when partial cooperation between source nodes is possible, by performing rate-splitting, message exchange, and superposition coding with proper power allocation at the source nodes, SNNC can achieve a strictly larger rate region than NNC. The gain comes from coherent combining at all the receiving nodes.
\end{abstract}

\section{INTRODUCTION}

The standard compress-and-forward $(\mathrm{CF})$ relaying strategy [1, Theorem 6] provides the destination node(s) with a noisy yet structured observation (compression) of its received signal via the use of an independent codebook. The CF strategy can be described as follows: the source transmits an independent short message in each time slot via random coding; the relay performs compression based on Wyner-Ziv binning; the destination performs block-by-block forward successive decoding (first decoding the bin index and then the message). The rate achieved by $\mathrm{CF}$ in the classical 3-node $\mathcal{S}-\mathcal{R}-\mathcal{D}$ relay model can be written as [1]

$$
\begin{gathered}
R<\max I\left(X_{s} ; Y_{d} \hat{Y}_{r} \mid X_{r}\right), \\
\text { subject to } I\left(Y_{r} ; \hat{Y}_{r} \mid X_{r} Y_{d}\right) \leq I\left(X_{r} ; Y_{d}\right),
\end{gathered}
$$

or, equivalently [3],

$$
\begin{aligned}
& R<\max \min [ I\left(X_{s} ; Y_{d} \hat{Y}_{r} \mid X_{r}\right), \\
&\left.I\left(X_{s} X_{r} ; Y_{d}\right)-I\left(Y_{r} ; \hat{Y}_{r} \mid X_{s} X_{r} Y_{d}\right)\right]
\end{aligned}
$$

where the maximization is over all distributions that can be factorized as

$$
p\left(x_{s}\right) p\left(x_{r}\right) p\left(\hat{y}_{r} \mid y_{r}, x_{r}\right) p\left(y_{r}, y_{d} \mid x_{s}, x_{r}\right) .
$$

The use of Wyner-Ziv binning at the relay makes CF nontrivial to extend to multiple relays [2].

The quantize-map-and-forward (QMF) protocol proposed in [4], which utilizes symbol-by-symbol scalar quantization at the relay and joint decoding at the destination, has been proved to be approximately optimal (within a constant gap to the cut-set bound) for unicast layered networks with multiple relays, and for non-layered unicast networks via time extension. The recently proposed noise network coding (NNC) protocol [5] can be regarded as an extension of QMF. NNC, which performs repetition coding at source nodes, compression without using Wyner-Ziv binning (vector quantization) at relays, and simultaneous joint message and compression index decoding at destinations, can be easily extended to multiplesource and/or multiple-relay scenarios. Contrary to CF where one large message is first partitioned into many blocks and then transmitted, NNC encodes the large message directly and transmits over all the time slots, each with an independent codebook. After all the transmissions are completed, the destination decodes the large message and all the compression indexes jointly. NNC has been shown in [5] to recover the rate region achieved by $\mathrm{CF}$ in the classical 3-node relay model, and outperform $\mathrm{CF}$ in the two-way relay channel, the interference relay channel, and the multiple-relay channel discussed in [2].

$\mathrm{Wu}$ and $\mathrm{Xie}$ have pointed out in [6] that the superiority of NNC over CF is due to the postponed decoding process, rather than the large-message repetition coding or the joint decoding of message and compression indexes. The benefit of the postponed decoding has also been discovered by [7] in which an extended $\mathrm{CF}$ has proposed by utilizing similar encoding process as in NNC at the source and the relay, but backward joint decoding at the destination.

As pointed out in [6], by using classical short-message encoding at the source node, compression without binning at relay nodes, and block-by-block backward decoding (either successively or jointly) at the destinations, one can achieve the same rate as NNC, as long as the relaying signal can be treated as noise at destinations. This new scheme is coined shortmessage noisy network coding (SNNC) in [8]. A formal rateequivalence proof of $\mathrm{NNC}$ and $\mathrm{SNNC}$ for the single-source multiple-relay network was given in [6]. A simpler alternative rate-equivalence proof has been given for the single-source single-relay network [8] and the single-source multiple-relay network [9]. The rate equivalence can also be established for the multiple-source multiple-relay network [10].

Using SNNC instead of regular NNC reduces encoding delay while still allowing for extensions to multiplesource/relay/destination networks. In addition, the short- 
message transmission facilitates source cooperation, in the presence of backhaul or a conferencing channel between source nodes.

In the rest of this paper, we show that when partial cooperation between source nodes is possible, SNNC can achieve a strictly larger rate region than $\mathrm{NNC}$ by performing ratesplitting [11], message exchanging, and superposition coding with proper power allocation at source nodes.

\section{SNNC With PARTial Source COOPERATION}

We consider a multiple-source single-relay network ${ }^{1}$ where two source nodes intend to multicast $W_{1}$ and $W_{2}$ to both destinations via the help of a full-duplex relay node, as shown in Fig. 1. The two source nodes can exchange information via orthogonal backhaul channels at rate $C_{12}$ and $C_{21}$ bits per channel use, respectively. Define $\gamma_{i j}=\frac{g_{i j}^{2} P_{i}}{\sigma_{j}^{2}}$ as link SNR, the received signal at relay and destination nodes can be written as follows,

$$
\begin{aligned}
& Y_{1}^{(n)}=\sqrt{\gamma_{11}} X_{1}^{(n)}+\sqrt{\gamma_{21}} X_{2}^{(n)}+\sqrt{\gamma_{r 1}} X_{r}^{(n)}+Z_{1}^{(n)}, \\
& Y_{2}^{(n)}=\sqrt{\gamma_{21}} X_{1}^{(n)}+\sqrt{\gamma_{22}} X_{2}^{(n)}+\sqrt{\gamma_{r 2}} X_{r}^{(n)}+Z_{2}^{(n)}, \\
& Y_{r}^{(n)}=\sqrt{\gamma_{1 r}} X_{1}^{(n)}+\sqrt{\gamma_{2 r}} X_{2}^{(n)}+Z_{r}^{(n)},
\end{aligned}
$$

where $Z_{i}[k]$ : i.i.d. $\mathcal{N}(0,1), \frac{1}{n} \sum_{k=1}^{n} E\left(X_{i}^{2}[k]\right) \leq 1, \quad i=$ $1,2, r$. A similar network topology in Fig. 1 has also been studied in [12] for packet erasure networks with two unicast sessions, whereas in our work we focus on Gaussian networks with two multicast transmission.

In the case of finite-rate backhaul, message exchange is impossible using NNC (since this will require the backhaul rates to approach infinity as the transmission block number $B \rightarrow \infty)$. SNNC, which divides $W_{1}\left(W_{2}\right)$ evenly into $B$ short messages $W_{1, t}\left(W_{2, t}\right), t=1, \ldots, B$, facilitates message exchange and therefore can benefit from coherent combining gain when cooperation is done as follows:

1) rate splitting at source nodes:

$W_{1, t}=\left[W_{1 p, t}, W_{1 c, t}\right], W_{2, t}=\left[W_{2 p, t}, W_{2 c, t}\right]$;

2) $W_{1 c, t}$ and $W_{2 c, t}$ are exchanged via backhaul and then formulate $W_{c, t}=\left[W_{1 c, t}, W_{2 c, t}\right]$ before transmission $t$;

3) source node performs superposition coding:

$X_{1}=\sqrt{\alpha_{1}} X_{1 p}\left(W_{1 p, t}\right)+\sqrt{\bar{\alpha}_{1}} X_{c}\left(W_{c, t}\right)$ and

$X_{2}=\sqrt{\alpha_{2}} X_{2 p}\left(W_{2 p, t}\right)+\sqrt{\bar{\alpha}_{2}} X_{c}\left(W_{c, t}\right)$.

With proper power allocation $\alpha_{1}, \alpha_{2} \in[0,1]$, the received signals can be written as follows

$$
\begin{aligned}
Y_{1}^{(n)}=\sqrt{\alpha_{1} \gamma_{11}} X_{1 p}^{(n)}+\sqrt{\alpha_{2} \gamma_{21}} X_{2 p}^{(n)} & +\left(\sqrt{\overline{\alpha_{1}} \gamma_{11}}+\sqrt{\overline{\alpha_{2}} \gamma_{21}}\right) X_{c}^{(n)} \\
& +\sqrt{\gamma_{r 1}} X_{r}^{(n)}+Z_{1}^{(n)}, \\
Y_{2}^{(n)}=\sqrt{\alpha_{1} \gamma_{12}} X_{1 p}^{(n)}+\sqrt{\alpha_{2} \gamma_{22}} X_{2 p}^{(n)} & +\left(\sqrt{\alpha_{1} \gamma_{12}}+\sqrt{\alpha_{2} \gamma_{22}}\right) X_{c}^{(n)} \\
& +\sqrt{\gamma_{r 2}} X_{r}^{(n)}+Z_{2}^{(n)}, \\
Y_{r}^{(n)}=\sqrt{\alpha_{1} \gamma_{1 r}} X_{1 p}^{(n)}+\sqrt{\alpha_{2} \gamma_{2 r}} X_{2 p}^{(n)} & +\left(\sqrt{\alpha_{1} \gamma_{1 r}}+\sqrt{\alpha_{2} \gamma_{2 r}}\right) X_{c}^{(n)} \\
& +Z_{r}^{(n)} .
\end{aligned}
$$

\footnotetext{
${ }^{1}$ The source cooperation scheme proposed here applies to any network where a pair of source nodes are connected by conferencing links, although the corresponding achievable rate expressions have to be adjusted according to the message delivery requirement (unicast, multicast, etc.).
}

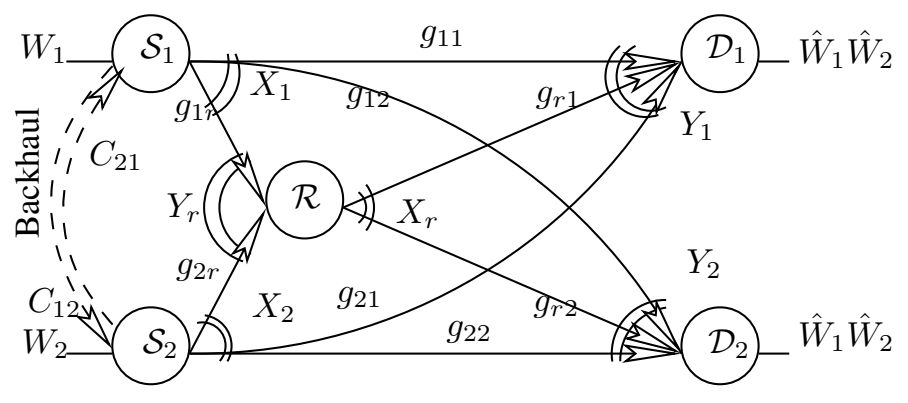

Fig. 1. Two source nodes $\mathcal{S}_{1}$ and $\mathcal{S}_{2}$, connected with backhaul (rate $C_{12}$ and $C_{12}$ ), multicast information $W_{1}$ at rate $R_{1}$ and $W_{2}$ at rate $R_{2}$ respectively to both destinations $\mathcal{D}_{1}$ and $\mathcal{D}_{2}$ through Gaussian channels, with aid from a full-duplex relay $\mathcal{R}$.

By message exchanging and superposition coding at source the nodes, we have transferred the 2 -source relay network described in (2) to a network with 3 independent sources $\left(X_{1 p}, X_{2 p}, X_{c}\right)$. The corresponding rate constraints $R_{1 p}, R_{2 p}, R_{c}$ can be obtained straightforwardly by applying SNNC/NNC results on independent sources.

Define $T=\left\{\mathcal{S}_{1 p}, \mathcal{S}_{2 p}, \mathcal{S}_{c}\right\}$ and $D=\left\{\mathcal{D}_{1}, \mathcal{D}_{2}\right\}$ with

$$
\begin{aligned}
X(T) & =\left\{X_{1 p} X_{2 p} X_{c}\right\}, \\
X\left(\mathcal{S}_{1 p}\right) & =X_{1 p}, X\left(\mathcal{S}_{2 p}\right)=X_{2 p}, X\left(\mathcal{S}_{c}\right)=X_{c}, \\
Y\left(\mathcal{D}_{1}\right) & =Y_{1}, Y\left(\mathcal{D}_{2}\right)=Y_{2}, Y(D)=\left\{Y_{1} Y_{2}\right\} .
\end{aligned}
$$

Let $S, S^{c}$ be any pair of complementary subsets of $T$, i.e., $S \cup S^{c}=T$ and $S \cap S^{c}=\emptyset$, we can define the following rate

$$
R(S)=\min _{d \in D} \max \left\{\begin{array}{c}
I(X(S) ; Y(d) \mid Q), \\
\min \left[I\left(X(S) ; \hat{Y}_{r} Y(d) \mid X\left(S^{c}\right) X_{r} Q\right),\right. \\
I\left(X(S) X_{r} ; Y(d) \mid X\left(S^{c}\right) Q\right) \\
\left.-I\left(\hat{Y}_{r} ; Y_{r} \mid X(T) X_{r} Y(d) Q\right)\right]
\end{array}\right\} .
$$

By applying the results of SNNC/NNC [5] into (3), the achievable rate region of SNNC with partial source cooperation can therefore be described as the union of all rate pairs $\left(R_{1}, R_{2}\right)$ that satisfy

$$
\left\{\begin{array}{l}
R_{1}=R_{1 p}+R_{1 c}, R_{2}=R_{2 p}+R_{2 c}, \\
0 \leq R_{1 c} \leq C_{12}, 0 \leq R_{2 c} \leq C_{21}, \\
0 \leq R_{1 p}<R\left(\left\{\mathcal{S}_{1 p}\right\}\right), \\
0 \leq R_{2 p}<R\left(\left\{\mathcal{S}_{2 p}\right\}\right), \\
R_{1 c}+R_{2 c}<R\left(\left\{\mathcal{S}_{c}\right\}\right), \\
R_{1 p}+R_{2 p}<R\left(\left\{\mathcal{S}_{1 p}, \mathcal{S}_{2 p}\right\}\right), \\
R_{1 p}+R_{1 c}+R_{2 c}<R\left(\left\{\mathcal{S}_{1 p}, \mathcal{S}_{c}\right\}\right), \\
R_{2 p}+R_{1 c}+R_{2 c}<R\left(\left\{\mathcal{S}_{2 p}, \mathcal{S}_{c}\right\}\right), \\
R_{1 p}+R_{2 p}+R_{1 c}+R_{2 c}<R\left(\left\{\mathcal{S}_{1 p}, \mathcal{S}_{2 p}, \mathcal{S}_{c}\right\}\right),
\end{array}\right.
$$

with the union taken over all joint distributions that can be factorized as

$$
p(q) p\left(x_{1 p} \mid q\right) p\left(x_{2 p} \mid q\right) p\left(x_{c} \mid q\right) p\left(x_{r} \mid q\right) p\left(\hat{y}_{r} \mid x_{r}, y_{r}, q\right) .
$$

By setting $Q=\emptyset$ and $\hat{Y}_{r}=Y_{r}+\hat{Z}_{r}$ with $\hat{Z}_{r} \sim \mathcal{N}\left(0, \sigma^{2}\right)$, all these mutual information constraints in (4) can be translated into $\mathcal{C}(x)=\frac{1}{2} \log _{2}(1+x)$ expressions which are functions of $\left(\alpha_{1}, \alpha_{2}, \sigma^{2}\right)$. We then introduce notations $I_{A}, \ldots, I_{G}$ to indicate 
these constraints (depending only on $\alpha_{1}, \alpha_{2}, \sigma^{2}$ and link SNR $\gamma_{i j}$ ) as follows

$$
\begin{aligned}
& I_{A}=R\left(\left\{\mathcal{S}_{1 p}\right\}\right), I_{B}=R\left(\left\{\mathcal{S}_{2 p}\right\}\right), I_{C}=R\left(\left\{\mathcal{S}_{c}\right\}\right), \\
& I_{D}=R\left(\left\{\mathcal{S}_{1 p}, \mathcal{S}_{2 p}\right\}\right), I_{E}=R\left(\left\{\mathcal{S}_{1 p}, \mathcal{S}_{c}\right\}\right), \\
& I_{F}=R\left(\left\{\mathcal{S}_{2 p}, \mathcal{S}_{c}\right\}\right), I_{G}=R\left(\left\{\mathcal{S}_{1 p}, \mathcal{S}_{2 p}, \mathcal{S}_{c}\right\}\right) .
\end{aligned}
$$

Remark 1: Given $\left(\alpha_{1}, \alpha_{2}, \sigma^{2}, \gamma_{i j}\right)$, we can conclude that $I_{A} \leq I_{E}$ and $I_{C} \leq I_{E}$ but NOT $I_{E} \leq I_{A}+I_{C}$ due to the minimization in $R(S)$. Similarly, we have $\max \left\{I_{D}, I_{E}, I_{F}\right\} \leq I_{G}$ but NOT $I_{G} \leq I_{A}+I_{F}, I_{G} \leq I_{B}+I_{E}$, or $I_{G} \leq I_{C}+I_{D}$.

After performing Fourier-Motzkin elimination over (4), the achievable rate region of SNNC with partial source cooperation for the system (2) is the union of all rate pairs $\left(R_{1}, R_{2}\right)$ that satisfy $R_{1} \geq 0, R_{2} \geq 0$, and

$$
\left\{\begin{aligned}
R_{1} & <\min \left\{I_{A}+C_{12}, I_{A}+I_{C}, I_{E}\right\} \\
R_{2} & <\min \left\{I_{B}+C_{21}, I_{B}+I_{C}, I_{F}\right\} \\
R_{1}+R_{2} & <\min \left\{I_{G}, I_{A}+I_{B}+I_{C}, I_{E}+I_{B}, I_{F}+I_{A},\right. \\
& \left.I_{D}+I_{C}, I_{D}+C_{12}+C_{21}, \frac{I_{D}+I_{E}+I_{F}}{2}\right\} \\
2 R_{1}+R_{2} & <I_{D}+I_{E}+C_{12}, \\
R_{1}+2 R_{2} & <I_{D}+I_{F}+C_{21},
\end{aligned}\right.
$$

where the union operation is taken over all $\alpha_{1}, \alpha_{2} \in[0,1]$ and $\sigma^{2} \in(0, \infty)$.

The standard CF scheme, which uses short messages, can also benefit from source cooperation in the same way as in SNNC. When a single Wyner-Ziv binning process is used at the relay node, by generalizing the results in [13] for the scenario with a single relay and two independent sources, the achievable rate region of the standard $\mathrm{CF}$ with partial source cooperation can be describe in the same way as in (4) but with

$$
R(S)=\min _{d \in D} I\left(X(S) ; \hat{Y}_{r} Y(d) \mid X\left(S^{c}\right) X_{r} Q\right),
$$

subject to

$$
\max _{d \in D} I\left(\hat{Y}_{r} ; Y_{r} \mid X_{r} Y(d) Q\right) \leq \min _{d \in D} I\left(X_{r} ; Y(d) \mid Q\right),
$$

where the distribution is partitioned as in (5).

Remark 2: The achievable rates by $\mathrm{CF}$ with cooperation will in general be smaller than the rates obtained by SNNC. For scenarios with multiple sources and multiple destinations (e.g. interference relay channel), decoding at different destinations require different level of side information, which is impossible for standard CF where a single Wyner-Ziv binning process is used. However for NNC/SNNC, as pointed out by [6], there is room to play with these different requirements and find a trade-off. Besides, successful decoding of the same binning index is confined by the weakest channel, as demonstrated in (8). The gain of NNC (and thus SNNC) over standard $\mathrm{CF}$ in terms of sum-rate has been demonstrated in [5] for the two-way relay channel and the interference relay channel. In addition, SNNC with source cooperation can be easily extended to multiple-source multiple-relay scenarios, which is not the case for standard CF due to to the presence of Wyner-Ziv binning.

\section{NNC With PARTIAL SOURCE COOPERATION}

With the finite-rate backhual, NNC does not allow cooperation as the number of message blocks $B$ goes to infinity, since one large message is transmitted in multiple blocks, and hence the common message should be identified to start with, in the first block already. However, NNC can still benefit from the backhaul in the following two ways: compression forwarding or message exchange.

\section{A. NNC with Compression Forwarding}

At transmission block $t=1, \ldots, B$, source node $\mathcal{S}_{1}$ forwards a compression $X_{s 1, t}$ at rate $C_{12}$ bits per channel use via backhaul to $\mathcal{S}_{2}$, and $\mathcal{S}_{2}$ forwards $X_{s 2, t}$ to $\mathcal{S}_{1}$ as follows,

$$
\begin{aligned}
& X_{s 1, t}=f_{1}\left(W_{1}, X_{s 1}^{t-1}, X_{s 2}^{t-1}\right), \\
& X_{s 2, t}=f_{2}\left(W_{2}, X_{s 2}^{t-1}, X_{s 1}^{t-1}\right),
\end{aligned}
$$

where $X_{s 1}^{t-1}=\left[X_{s 1,1}, \ldots, X_{s 1, t-1}\right], X_{s 2}^{t-1}=\left[X_{s 2,1}, \ldots, X_{s 2, t-1}\right]$, $f_{1}(\cdot)$ and $f_{2}(\cdot)$ are some compression functions. At block $t+1$, $\mathcal{S}_{1}$ broadcasts $X_{1, t+1}$ which is determined based on $W_{1}$ and $X_{s 2, t}$. With compression forwarding through backhaul, source nodes essentially behave as a relay node to help the delivery of each other's messages, which fits well into the framework of $\mathrm{NNC}$ and therefore only some slight modification on the encoding/decoding process is needed for the extension. Although there are many different ways to design the compression functions, the optimal way is yet to be determined based on the available backhaul capacity, channel settings, and message delivery requirement. We will investigate the optimality of different compression forwarding methods for NNC via finiterate backhaul in future work.

Remark 3: With compression forwarding via backhaul, the transmitting signal $X_{1, t}\left(X_{2, t}\right)$ is a function of the large message $W_{1}\left(W_{2}\right)$, which is not known at the other source. Hence there is no coherent combining gain for NNC with compression forwarding, which is not the case for SNNC and $\mathrm{CF}$ where rate-splitting and message exchange is used instead with superposition coding.

To illustrate the gain of SNNC with partial source cooperation over $\mathrm{NNC}$ and $\mathrm{CF}$, we have plotted the achievable rate regions in Fig. 2 for an asymmetric channel setup of the system as in Fig. 1. The outer bound is obtained based on the genie-aided cut-set bound and the inner bound of NNC is obtained by compression forwarding as proposed in [14], where $X_{s 1, t}$ is used to convey a noisy version of $X_{1, t}$ to $\mathcal{S}_{2}$. Such noise observation is then used to generate a compression index as in the normal NNC strategy. With partial cooperation, SNNC achieves a strictly larger rate region than NNC with compression forwarding, and the gain comes from coherent combining at all the receiving nodes. $\mathrm{CF}$, on the other hand, performs better than NNC (even with compression forwarding) when coherent combining gain is large $\left(C_{12}=0.9\right)$, but worse than NNC (even without cooperation) when the asymmetric channel setting effect in (8) dominates the coherent combining gain $C_{21}=0.1$ ). The degradation of $\mathrm{CF}$ compared to SNNC and is due to the asymmetric channel setting which causes 


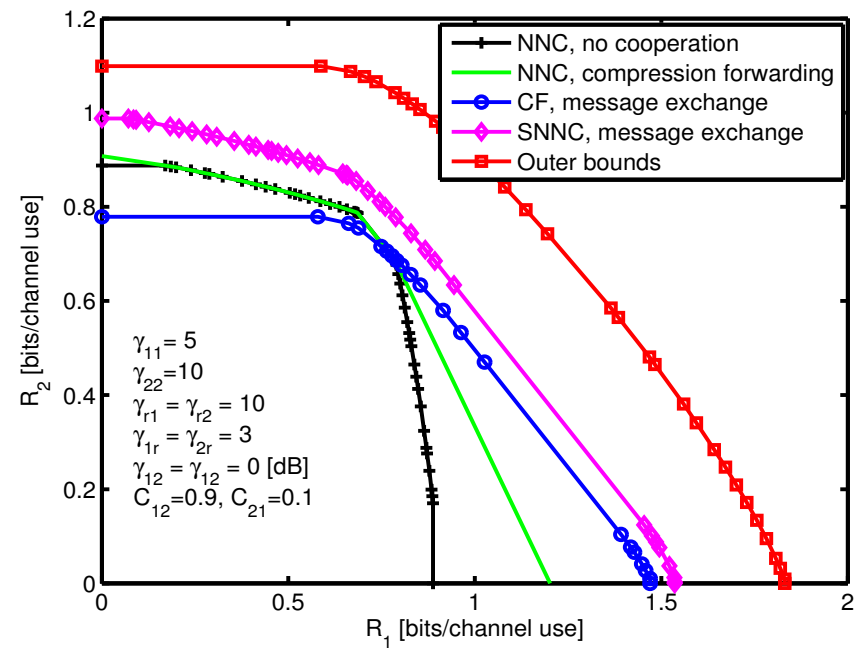

Fig. 2. Achievable rate regions of SNNC in the asymmetric channel setting with source conferencing rate $C_{12}=0.9$ and $C_{21}=0.1$ bits per channel use. NNC with no cooperation is plotted as reference.

different requirement on successive decoding of the WynerZiv binning index, as explained in Remark 2, see also [6].

\section{B. NNC with Message Exchange}

NNC with message exchange via backhaul can be done in a segment-by-segment fashion ${ }^{2}$ : partition the total message blocks into $K$ segments, each with $B$ blocks; during the first segment NNC is used without cooperation but the backhaul is used to exchange messages to be transmitted in segment 2 ; in segment $m>1$ three large messages $W_{1 p}, W_{2 p}, W_{c}$ are transmitted using superposition encoding. This however requires long memories and long encoding delay that grow with $n \times B$, which is not the case in the SNNC strategy.

On the other hand, as stated in [5], the achievable rate of NNC with finite $B$ and sufficiently long codeword length $(n \rightarrow$ $\infty$ ) for the classical 3-node relay channel is

$$
\begin{aligned}
& R_{B}<\frac{B-1}{B} R_{\infty}-\frac{I\left(\hat{Y}_{r} ; Y_{r} \mid X_{r}\right)}{B}, \\
& \text { where } R_{\infty}=\min \left\{I\left(X_{s} ; Y_{d} \hat{Y}_{r} \mid X_{r}\right),\right. \\
& \left.\qquad I\left(X_{s} X_{r} ; Y_{d}\right)-I\left(Y_{r} ; \hat{Y}_{r} \mid X_{s} X_{r} Y_{d}\right)\right\}
\end{aligned}
$$

is the rate of NNC when $B \rightarrow \infty$. Therefore NNC with segment-wise cooperation can achieve rate $R_{B}$ for all the $K$ segments except for the first one where no cooperation is done (hence a lower rate than $R_{B}$ ). The corresponding achievable rate for SNNC with the same number of message blocks is

$$
R_{B \times K}<\frac{B \times K-1}{B \times K+M} R_{\infty}-\frac{I\left(\hat{Y}_{r} ; Y_{r} \mid X_{r}\right)}{B \times K},
$$

where $M<<B \times K$ is the extra blocks to ensure the start of backward decoding [6]. As the cooperation block number $B$ cannot be very large due to memory constraint, NNC with cooperation will still incur a rate loss compared to SNNC.

\footnotetext{
${ }^{2}$ The cooperation for NNC will be done over message blocks within the same segment and no cooperation across segments.
}

Remark 4: In absence of memory and encoding delay constraints (i.e., $B \rightarrow \infty$ ), and introducing fading (known only at the receiving sides, either relay or destinations), NNC may actually be advantageous, as there will be many blocks conveying the same message, ergodic rates prevail while short messages, even with backward decoding are subject to outages. This issue will be investigated in the future work.

\section{CONClusions}

Allowing partial source cooperation, we have demonstrated that encoding delay and memory constraints can affect the achievable rate of regular NNC, and employing instead shortmessage NNC can provide significant gains and therefore can achieve a strictly larger rate region than NNC. In absence of encoding delay and memory constraints, however, NNC with message exchange can achieve almost the same rate as SNNC and therefore outperforms $\mathrm{NNC}$ with compression forwarding due to the coherent combining gain. The optimal scheme of compression forwarding for NNC and the optimal quantization/compression rate at relay nodes in wireless network with multiple destinations are yet to be studied in future work.

\section{ACKNOWLEDGMENTS}

This work was funded in part by the Swedish Governmental Agency for Innovation Systems (VINNOVA) and by the Swedish Research Council (VR). The work of S. Shamai has been supported by the Israel Science Foundation (ISF).

\section{REFERENCES}

[1] T. M. Cover and A. El Gamal, "Capacity theorems for the relay channel," IEEE Trans. Inf. Theory, vol. 25, pp. 572-584, Sep. 1979.

[2] G. Kramer, M. Gastpar, and P. Gupta, "Cooperative strategies and capacity theorems for relay networks," IEEE Trans. Inf. Theory, vol. 51, pp. 30373063, Sep. 2005.

[3] A. El Gamal, M. Mohseni, and S. Zahedi, "Bounds on capacity and minimum energy-per-bit for AWGN relay channels", IEEE Trans. Inf. Theory, vol. 52, pp. 1545-1561, Apr. 2006.

[4] A. S. Avestimehr, S. N. Diggavi, and D. N. C. Tse, "Wireless network information flow: a deterministic approach," IEEE Trans. Inf. Theory, vol. 57, pp. 1872-1905, Apr. 2011.

[5] S. H. Lim, Y.-H. Kim, A. El Gamal, and S.-Y. Chung, "Noisy network coding," IEEE Trans. Inf. Theory, vol. 57, pp. 3132-3152, May 2011.

[6] X. Wu and L.-L. Xie, "On the optimal compressions in the compressand-forward relay schemes," arXiv:1009.5959

[7] A. Raja and P. Viswanath, "Compress-and-forward scheme for relay networks: backword decoding and connection to bisubmodular flows," arXiv:1012.0416v3, Jun. 2012

[8] G. Kramer and J. Hou, "Short-message quantize-forward network coding," in Proc. of 8th Int. Workshop on Multi-Carrier Systems \& Sollutions, May 2011.

[9] G. Kramer and J. Hou, "On message lengths for noisy network coding," in Proc. of IEEE ITW, Oct. 2011.

[10] G. Kramer, "Progress on relaying and noisy network coding," Keynote lecture at Internation Zürich Seminar, Feb. 2012.

[11] B. Rimoldi and R. Urbanke, "A rate-splitting approach to the Gaussian multiple-access channel," IEEE Trans. Inf. Theory, vol. 42, pp. 364-375, Mar. 1996.

[12] W.-C. Kuo and C.-C. Wang, "On the capacity of 2-user 1-hop relay erasure networks - The union of feedback, scheduling, opportunistic routing, and network coding," in Proc. of IEEE ISIT, Aug. 2011.

[13] D. Gündüz, O. Simeone, A. J. Goldsmith, H. V. Poor, and S. Shamai, "Multiple multicasts with the help of a relay," IEEE Trans. Inf. Theory, vol. 56, pp. 6142-6158, Dec. 2010.

[14] J. Du, M. Xiao, M. Skoglund, and M. Médard, "Wireless multicast relay networks with limited-rate source-conferencing," IEEE J. Selected Areas in Commun., accepted. To appear in Sep. 2012. 